ELECCIÓN PURA, DESEMPEÑO SOCIOECONÓMICO Y PARTICIPACIÓN POLÍTICA: UNA EVALUACIÓN ALTERNATIVA

MARÍA STELLA BACCA ROSERO

UNIVERSIDAD SANTO TOMÁS DE AQUINO

FACULTAD DE CIENCIAS ECONÓMICAS

BOGOTÁ

2016 


\title{
ELECCIÓN PURA, DESEMPEÑO SOCIOECONÓMICO Y PARTICIPACIÓN POLÍTICA: UNA EVALUACIÓN ALTERNATIVA
}

\author{
MARÍA STELLA BACCA ROSERO
}

Trabajo para optar al título de Magister en Ciencias Económicas

Director

LUIS NELSÓN BELTRÁN MORA

Profesor titular

UNIVERSIDAD SANTO TOMÁS DE AQUINO

FACULTAD DE CIENCIAS ECONÓMICAS

BOGOTÁ

2016 
Elección pura, desempeño socioeconómico y participación política: una evaluación alternativa

\section{Pure choice, socioeconomic performance and political participation: an alternative evaluation}

\section{Resumen}

En esta investigación se evalúan dos afirmaciones sobre el proceso democrático tal como es concebido por la teoría de la elección pública (Public Choice) aplicada al caso colombiano en años recientes. En primer lugar, la tendencia a la polarización en partidos políticos antagónicos, como complemento o lado alternativo del concepto del votante medio. En segundo lugar, la interacción entre la participación política y los indicadores fiscales y sociales, resultado de la gestión de los gobiernos municipales. La polarización se evalúa con algunas herramientas estadísticas y un índice de concentración modificado de Herfindahl, mientras que la participación y los resultados del gobierno son representados por estructuras de ordenamiento y después comparados con un indicador de semejanza. Los ejercicios cuantitativos mostraron que la polarización, en el caso de Colombia, no se ha reforzado en los años transcurridos del siglo XXI, y que de modo paralelo ha tenido lugar una coincidencia positiva (aunque medio) entre la participación política y las variables representativas de la gestión pública.

\section{Palabras clave}

Elección colectiva, función de bienestar social, participación política, entidades territoriales.

Clasificación JEL: A16, C43, D6. 


\begin{abstract}
This research evaluates two statements about a democratic process as conceived by Public Choice theory, applied to Colombian case in recent years. In the first place, the tendency to polarization in antagonistic political parties, as a complement or alternative side of median voter concept. And second, the interaction between political participation and fiscal and social indicators results from local governments management. Polarization is evaluated through some statistic tools and a modified Herfindahl concentration index, whereas participation and government results are represented by ranking structures and next compared by a similarity indicator. Quantitative exercises showed that polarization in Colombia has not been reinforced along elapsed years of 21 nd Century, and in parallel way there is a positive (although slight) coincidence between political participation and public management representative variables.
\end{abstract}

\title{
Keywords
}

Public choice, social welfare function, political participation, local governments.

Clasification JEL: A16, C43, D6. 


\section{TABLA DE CONTENIDO}

Pág.

Introducción

1. Aspectos teóricos: Elección social, bienestar y teoría económica ortodoxa de la democracia

2. Propuesta metodológica 16

2.1 Dinámica de la polarización política 16

2.2 Participación política y desempeño socioeconómico 20

3. Resultados de la evaluación $\quad 27$

3.1 Cambios en el espectro político en Colombia 2002-2014 27

3.2 Cotejo entre participación política y gestión pública municipal 29

4. Conclusiones 35

Anexo. Municipios excluidos de la evaluación por sus

niveles atípicos de participación política en 2011

$\begin{array}{ll}\text { Referencias bibliográficas } & 40\end{array}$ 


\section{LISTA DE TABLAS}

Pág.

Tabla 1. Simulación de cálculo del indicador de semejanza

Tabla 2. Proporciones de votantes en el espectro político colombiano

Tabla 3. Indicadores de polarización política

Tabla 4. Cálculo de indicadores de semejanza 


\section{LISTA DE FIGURAS Y GRÁFICOS}

Pág.

Figura 1. Espectro de preferencias políticas en una distribución unimodal

Figura 2. Espectro de preferencias políticas en una distribución bimodal 


\section{Introducción}

Aunque el referente primordial de observación de la ciencia económica sea la valoración social de las instancias de producción, distribución y consumo de bienes y servicios, ya sea en el contexto del sistema de asignación de mercado o en formas alternativas a este, en las últimas décadas la investigación económica ha extendido su perspectiva de análisis a diversas temáticas de la interacción humana, buscando explicar los móviles racionales que le subyacen mediante modelos en los que la conducta del individuo sigue pautas semejantes a las del agente optimizador neoclásico.

En este mismo espectro de campos de análisis ha entrado la actividad política, en la cual se ponen de manifiesto las diferencias de enfoque entre lo que pretende el individuo elector o votante, y el individuo "político" o "representante", es decir, el agente que conduce (o debiera conducir) al debate los intereses de una colectividad respecto a sus necesidades públicas, aquellas que versan sobre bienes de no mercado, como la defensa y la seguridad, la libertad de expresión, el medio ambiente, entre muchos otros. Especial relevancia ha adquirido a este respecto la teoría de la elección pública (Public Choice), cuyo planteamiento central consiste en que la democracia formal, plasmada en los procesos electorales de gobernantes y corporaciones públicas, puede ser interpretada como un proceso competitivo entre caudillos que buscan el favor del elector, de modo tal que las preferencias de aquéllos se sobreponen a las de este último. Los idearios políticos, de modo similar a una mercancía, se acercan al consumidor medio (votante medio), y si este fenómeno ocurre de manera continua, bajo el supuesto de que las preferencias o simpatías políticas tienen una distribución unimodal (de forma acampanada), es de esperar una similitud cada vez mayor entre las 
posturas ideológicas y un agrupamiento de los electores alrededor de la preferencia del votante medio.

Frente al anterior planteamiento, la política colombiana ha mostrado en lo corrido del siglo XXI manifestaciones de extremismo o polarización que discrepan del agrupamiento de electores alrededor de una postura media, por lo que es de particular interés la utilización de herramientas de evaluación cuantitativa que provean una respuesta al interrogante de si las preferencias de los ciudadanos, tal como han sido expresadas en las urnas, sustentan el argumento de una polarización reforzada en las posiciones ideológicas entorno al modelo deseable de país. En consonancia con ello, la investigación acomete esta tarea.

Así mismo, cabe cuestionar o replantear, a la luz de conceptos como el de las expectativas racionales, la naturaleza estática o pasiva del elector frente a la función de utilidad del candidato o caudillo en la argumentación desarrollada por la escuela Public Choice. El cuestionamiento tiene su origen en las diferencias tan notables en los logros de coberturas sociales y de indicadores fiscales entre las entidades territoriales en Colombia, sobre cuyo desempeño se sugiere en el presente documento que puede estar asociado a rasgos intrínsecos de los procesos electorales, como la participación, el número de candidatos, la concentración de votos $u$ otras variables que pueden representar de manera indirecta características de la agregación de preferencias individuales. En consecuencia, se expone una visión alterna del elector como agente activo, capaz de incidir sobre los contenidos programáticos ofrecidos por los candidatos en el desarrollo de un proceso electoral. De este modo, se somete a validación la hipótesis de sintonía o armonización entre los niveles de participación política y los logros de los gobiernos municipales a través de algunas variables de insumo para la asignación de recursos del Sistema General de Participaciones (SGP). De ser este el caso, el de una interacción positiva entre ambos tipos de variables, se 
dispondrá de una evidencia para la formulación de planteamientos alternos o heterodoxos respecto a la visión neoclásica de los procesos políticos. 


\section{Aspectos teóricos: Elección social, bienestar y teoría económica ortodoxa de la democracia}

La sociedad de finales del siglo XX y los albores del siglo XXI se cataloga como la sociedad de la información y las comunicaciones. El instrumental tecnológico de la era digital permite, a un costo bajo, disponer de información de muy variada naturaleza que años atrás hubiese sido muy dispendioso adquirir. En la medida que la información es demandada y apropiada, la civilización se transforma, modelando individuos cuyo espectro de aspiraciones y búsquedas supone nuevos retos para asumir por parte de las ciencias sociales. Aunque sigan predominando en el ideario colectivo los conceptos de riqueza y poder, propios mas no exclusivos del capitalismo occidental, la forma de aproximación a los mismos reviste modificaciones notables derivadas en parte una mayor conciencia por parte de distintos grupos poblacionales sobre su papel en la sociedad y su capacidad de incidencia sobre las decisiones públicas. La convicción de que gobiernos legítimamente populares puedan responder mejor a las demandas sociales que los modelos autoritarios motiva a examinar los alcances efectivos de la participación política sobre el bienestar en sus distintas acepciones o componentes. Muy atrás ha quedado en el discurso del desarrollo la idea antigua de la identificación del bienestar con la simple generación y acumulación de producción.

Desde distintas ópticas la teoría económica representa la transición analítica desde las elecciones individuales hacia las colectivas. Por ejemplo, la nueva macroeconomía clásica, ligada a la Escuela de Chicago, utiliza la noción del agente representativo (Lucas, 1975) para modelar variables agregadas como si detrás de estas hubiese un único individuo o actor cuyo comportamiento optimizador se rigiera por los postulados de la microeconomía. Esta simplificación del comportamiento social trae implícito el supuesto de que las propiedades de la 
elección individual son enteramente replicables a nivel colectivo, sin un examen de consistencia frente a eventuales contradicciones o frente a atributos distintivos del conglomerado social frente al individuo. El concepto de agente representativo también se utiliza para representar resultados agregados derivados de comportamientos diferenciados por grupos de agentes. Es el caso del productor que, ante un alza en el precio de su producto, puede interpretar que se trata de un alza en el nivel general de precios o de un alza en el precio relativo del producto en cuestión. Si se inclina por lo segundo, se incrementará la oferta de la economía, con el riesgo de haber incurrido en una percepción equivocada. Implícitamente puede interpretarse que en la situación mencionada ha acontecido un proceso de decisión grupal en el que los comportamientos individuales se "suman". Quienes percibieron un alza general de precios generan un incremento nulo de su oferta, y quienes percibieron un alza relativa generan un incremento mayor de cero. Según el peso de cada agente en la economía, la modificación en el nivel de oferta agregada reflejará una decisión colectiva.

Una segunda forma de aproximarse a la traducción del interés individual en interés colectivo consiste en examinar rasgos de las preferencias individuales que mediante un determinado procedimiento señalen las pautas de acción racionales por parte de la colectividad. Esta ruta de acción tuvo origen en el análisis microeconómico, pero como tal terminó constituyéndose en una línea de estudio propia: la economía del bienestar. El propósito esencial de esta sub-disciplina es la construcción de una función de bienestar social, es decir, un mecanismo de agregación de las utilidades o bienestares de los individuos de un colectivo que represente la magnitud de la utilidad o bienestar del conjunto. Desde la lectura microeconómica, la función de bienestar social tendría que reflejar una estructura de preferencias que aglutine el efecto de todas las preferencias individuales. 
En términos de Lange (1974), si las funciones de utilidad individual para un conjunto de $\mathrm{n}$ personas son:

$$
u^{(1)}, u^{(2)}, \ldots, u^{(n)}
$$

entonces la función de bienestar social, indistintamente de su forma funcional específica (aditiva, multiplicativa, logarítmica, exponencial, otra), es una expresión del tipo:

$$
u=f\left(u^{(1)}, u^{(2)}, \ldots, u^{(n)}\right)
$$

susceptible de variar, dada alguna restricción presupuestaria o de dotaciones de riqueza, hasta un punto en el que no sea posible incrementar la utilidad de un individuo sin reducir la utilidad de otro. Es decir, hasta alcanzar un óptimo paretiano.

Sobre este ejercicio teórico caben diversas observaciones. La primera concierne a la eliminación de las llamadas comparaciones interpersonales, por las cuales la asignación o reducción marginal de una cuantía monetaria o de riqueza real afecta en cuantías distintas la utilidad de individuos distintos (por ejemplo, un pobre contra un rico). Un modelo que contemplara este tipo de distinciones podría calificarse de arbitrario, en la medida que no formule un sustento racional para tal postulado. La optimización de una función de bienestar, bajo los atributos convencionales de la función de utilidad (convexidad, monoticidad), contempla que en el margen las utilidades de todos los individuos se equiparen. De no ser ese el caso, existiría siempre espacio para la mejora aditiva mediante la redistribución de recursos o riqueza. 
La segunda observación relevante guarda un vínculo muy fuerte con la primera, y alude a la cuantificación de la utilidad. La elección de alguna variable en el contexto empírico que represente la utilidad requeriría de un alto grado de consenso, además de una incidencia efectiva en las decisiones de política económica. Dado que una eventual cuantificación no posee ninguno de los citados atributos, se propende por descartarla en el proceso de construcción de la función de bienestar.

Algunas elaboraciones teóricas, no obstante, sin necesidad de seleccionar una unidad de medida contemplaron la cuantificación, y derivada de ella, la comparabilidad. Un ejemplo fue el caso de las intensidades divergentes dentro del modelo de Edgeworth (1879), que dio lugar a su cálculo hedónico. En él, cada individuo es afectado de distinta forma por un estímulo común (de igual magnitud), de modo que quien sienta mayor placer o mayor dolor dará una valoración más elevada al fenómeno que suscite una decisión de su parte. Los individuos de mayor "sensibilidad" reciben en esta elaboración una ponderación mayor que los individuos poco "sensibles".

La tercera observación relevante remite a la limitante deducida por Arrow (1974) sobre el proceso de elección, y dio lugar al conocido Teorema de la Imposibilidad. A partir de una serie de atributos deseables para la elección agregada, y consistentes con las estructuras de preferencia individual, Arrow concluye que las preferencias colectivas necesariamente coincidirán con la estructura de (al menos) un agente individual, con lo cual se quiebra el principio de no imposición. En lenguaje político, esto se traduce en que un esquema de agregación racional de elecciones forzosamente se traduce en una dictadura, no en el sentido de que el poder sea "tomado" o "cedido", sino en el sentido de que el colectivo social se comporta exactamente como lo haría (al menos) uno de sus individuos si este tuviese que decidir por el colectivo. De hecho, este resultado guarda una 
reveladora similitud con elaboraciones posteriores de fundamento microeconómico aplicado al orden macro, como el modelo de Blanchard (1989), en el cual la elección óptima de consumo y ahorro provista en el escenario convencional del mercado coincide con la elección que habría tomado una figura de planificador central, quien utiliza precisamente una función de bienestar para resolver el problema de optimización.

Hasta aquí han sido considerados dos planteamientos centrales alrededor del proceso de elección: uno en el que el comportamiento del todo (del colectivo) se asemeja cualitativamente al de la parte (el caso del agente representativo), y otro en el que la elección grupal es calificada de insatisfactoria precisamente por asemejarse en su estructura interna a la de al menos un individuo. Sin embargo, no se ha hecho apreciación alguna sobre el contenido del conjunto de elección. Lange (1974) recurre a una función de valor social elaborada a partir de funciones de utilidad individual, señalando que en el argumento de estas últimas no solo se hallan los bienes objeto de adquisición en el mercado, sino elementos carentes de valoración social explícita, como el ocio, la sensación de seguridad, el atractivo de la ocupación ejercida o el prestigio. En cambio, los modelos neoclásicos soportados en microfundamentos suelen estar restringidos al conjunto de transacciones ligadas a la dinámica del producto.

Cabe argumentar, entonces, que una teoría integral de la elección social debería aplicar especial énfasis sobre el modo como las decisiones relativas a los bienes de no-mercado se asemejan o discrepan de las decisiones de naturaleza mercantil. Incluso en este cometido deben diferenciarse las iniciativas emprendidas para asignarle valoración a los fenómenos propios del entorno o contexto en el que se desenvuelve la actividad económica (el ambiente, la cultura, el sistema político) del análisis propiamente dicho de la mecánica de la elección. Al respecto, González (1998) recuerda que en el trasfondo de la argumentación de 
Arrow se encuentra un rechazo a la distinción entre elecciones económicas y elecciones políticas, en el sentido de que estos procesos son manifestaciones de un concepto más amplio, la elección social colectiva.

La teoría microeconómica básica considera al individuo como ejecutor de un proceso optimizador sobre un conjunto acotado (subconjunto de un universo) y sujeto a restricciones. En la elección óptima de un consumidor son descartados los fenómenos sobre los que este incide por medios distintos a su poder adquisitivo, y este último también aparece como exógenamente determinado. Claramente, un intento de reformular el proceso de optimización de modo tal que agregue o incorpore las condiciones de entorno exige que el individuo exceda su ámbito singular de elección para adentrarse en la mecánica de la acción política. No necesariamente los ingresos, precios o condiciones del entorno pueden asumirse como "justos". Para propósitos del bienestar de una comunidad, el resultado estándar del equilibrio de los mercados es poco diciente. El propio Arrow, coautor del modelo de equilibrio neoclásico (modelo Arrow-Debreu), y quienes citado por González (1998), señala que los mercados no atienden al objetivo del bienestar. Como muestra acertadamente Sen (1976), un óptimo en la asignación de recursos puede ocurrir simultáneamente con una sociedad indignante, en la que por la condición de optimalidad de Pareto, no pueda mejorar la situación de los indigentes sin afectar a los individuos ricos.

Si las preferencias individuales por los bienes y servicios de mercado varían en su intensidad (reflejada en los parámetros de la función de utilidad), mucho mayor es la divergencia o disparidad entre los "estados deseables" para el orden social según la concepción de cada persona, hasta el punto de que tales diferencias conducen a conflictos. Un estado deseable es un único caso dentro del universo de eventuales o potenciales "estados del mundo", "estados de cosas" o "estados sociales" alrededor de los cuales se pretende formular la función de bienestar 
social. En cada uno de ellos se refleja tanto la situación del individuo que posee una aspiración o ideal, como la de sus semejantes dentro del colectivo. Por ende, es aquí donde entra la democracia como categoría de análisis. Esta, como manifestación empírica (aunque se dirá que muy imperfecta respecto de sus propios paradigmas) de los procesos de elección, puede proveer algunos elementos para abordar el problema central de la economía del bienestar sobre la agregación de preferencias. Por ejemplo, los procesos democráticos remiten a una categoría con poco o insuficiente tratamiento en la teoría económica, como lo es la comunicación dirigida a la comprensión de contenidos o la generación de acuerdos entre sujetos (Muguerza, 1992). Desde otra óptica, la democracia opera como mecanismo de equilibrio entre ideologías revestidas con la forma de "mercaderías políticas" (Quesada, 1992). De esta forma quedan planteadas dos conceptualizaciones acerca del sistema democrático: una, como escenario para el debate y las propuestas de corrección, superación o mitigación de las desigualdades del régimen capitalista vigente, considerando los alcances del mercado como institución; y otra, como uno entre muchos tipos de mecanismos ideados para generar decisiones que afectan a todos los miembros de una sociedad.

González (1992) afirma, no obstante, que parece existir la tendencia a que, luego de la extinción de algún régimen totalitario o dictatorial, los ideales populares de renovación democrática se diluyan paulatinamente, para ser reemplazados por simples enfoques de gestión, es decir por formas específicas de administrar el sistema capitalista imperante. Aparte de ello, para los participantes en política, más que la identificación argumentativa con los electores, priman variables como el éxito personal (prestigio, poder) y el oportunismo, de lo cual es muestra la preponderancia del carisma y la capacidad de expresión en los medios de comunicación. 
En lo que respecta a las propuestas políticas de los programas actuales, su escaso o ambiguo contenido ha llevado a que las diferencias ideológicas entre partidos sean mínimas, a menudo restringidas a cuestiones de procedimiento o de guarismos (grados de intervención, metas planteadas, etc.). Esta situación se acompaña de un proceso de atomización que fue predicho por Dahl (1956, citado por González, 1992) bajo el concepto de "pluralismo de minorías", es decir movimientos políticos con poco favor popular, aunque suficiente para ejercer representatividad, con alta flexibilidad para crear alianzas según el interés específico pretendido en una coyuntura dada (es decir, la manifestación del oportunismo).

Para brindar una comprensión más formal y menos descriptiva de tales fenómenos, irrumpió la teoría del Public Choice como forma de "encasillar" o "moldear" la acción política dentro del análisis económico, tal como ha acontecido con otras realidades desligadas del escenario de mercado. En esta corriente doctrinal (Escuela de Virginia), los objetivos pretendidos por el público y cuya consecución o logro se ha encomendado al gobierno, se convierten en objetivos alternativos para la asignación de recursos. En la medida que los individuos procuran alguna interlocución con quienes aspiran a ser sus representantes, de la cual surgen relaciones de intercambio, se valida la noción renovada de la economía como "teoría general del comportamiento y la interacción social" (Becker, 1977. Citado por González, 1992). Queda reiterado en este enfoque el concepto del homo oeconomicus como agente maximizador de utilidad en todos los ámbitos de su existencia.

De esta forma, a la luz de esta doctrina, los estamentos colectivos, como los partidos políticos o los cuerpos colegiados (Asambleas, Concejos, Congreso) son sencillamente instrumentos que recogen pretensiones surgidas de una racionalidad individualista, tanto del elector como la de quien lo representa. Esta 
división o escalamiento entre el individuo raso y quien transmite y divulga (a menudo en forma muy escasa) sus intereses bajo la forma de discurso político, lo que muestra es que la igualdad formal de derechos se contrapone a una desigualdad respecto a la influencia ejercida efectivamente sobre la acción de gobierno, más allá del simple acto de la votación. Los móviles propios del político (renta, influencia, prestigio) hacen las veces de argumentos en su función de utilidad individual. Desde la lógica del costo-beneficio, la adquisición de información sobre plataformas políticas-costosa en tiempo, dedicación y atención por parte del votante excede en valor (subjetivo) al beneficio consistente en que su voto individual, con una probabilidad dada, conduzca a decidir una elección. Esta argumentación, denominada "paradoja de la no votación" (Loyola, 2011) hace las veces de respaldo para el ejercicio de la abstención, no entendida como señal de apatía sino como conducta eminentemente racional. En un sentido extremo, la paradoja conduce a que, si cada votante por separado tuviera el mismo raciocinio de costo-beneficio, nadie votaría. Pero esto, a su vez haría que solamente una persona que votara decidiese el resultado de una elección, maximizando su beneficio.

Una herramienta gráfica de simplificación del tipo de análisis efectuado por el Public Choice es la distribución de preferencias de los electores sobre el espectro de ideologías, desde la extrema izquierda hasta la extrema derecha. En la siguiente figura, el eje coordenado horizontal muestra los "grados ideológicos" como una variable continua, y el eje vertical muestra la proporción de electores afines a cada grado, de modo análogo a una función de densidad de probabilidad. 
Figura 1. Espectro de preferencias políticas en una distribución unimodal

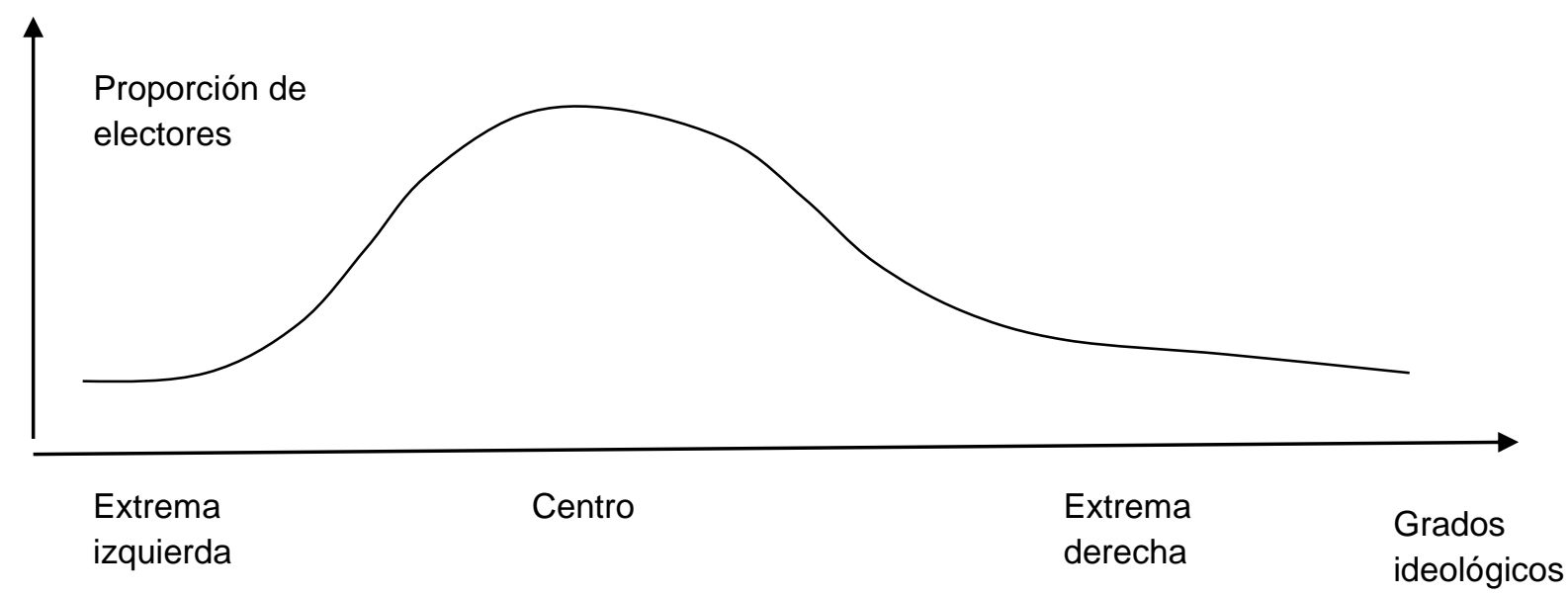

La proposición relativa a la forma acampanada alude al principio del votante medio, por el cual resulta atractivo para las organizaciones políticas situadas en alguno de los extremos de la figura captar la atención de votantes más próximos a la posición de centro. Esto incentivará el incremento de "ofertas políticas" dirigidas al votante medio. No obstante, el desplazamiento hacia el centro del espectro no es gratuito: paulatinamente, significará insatisfacción y eventual abandono por parte de los seguidores más radicales, hasta un punto en el cual, siguiendo el enfoque marginalista, los votos perdidos en la zona "radical" del espectro (izquierda o derecha) sean iguales a los ganados en la zona central. No necesariamente el proceso culmina desplazándose hasta la moda (valor más elevado, de mayor frecuencia) de la distribución. Puede culminar antes, debido a que sobre el votante medio concurren múltiples ofertas, y la probabilidad de ganar el favor de este votante se sopesa contra la probabilidad de pérdida de seguidores radicales. 
Un escenario de propuestas programáticas difusas, que para el votante medio fuesen muy difíciles de diferenciar, es propio de la zona media de la distribución de votantes antes descrita, pero es factible imaginar otros patrones gráficos, como uno con elevado grado de confrontación o polarización. Cabría suponer que en un escenario bimodal o multimodal, ante una mayor dificultad de aproximarse a los votantes cercanos en el espectro, la oferta de "mercadería política" puede verse sustituida por elementos extra-programáticos, como dádivas o promesas de retribución futura a individuos o grupos de electores.

Figura 2. Espectro de preferencias políticas en una distribución bimodal

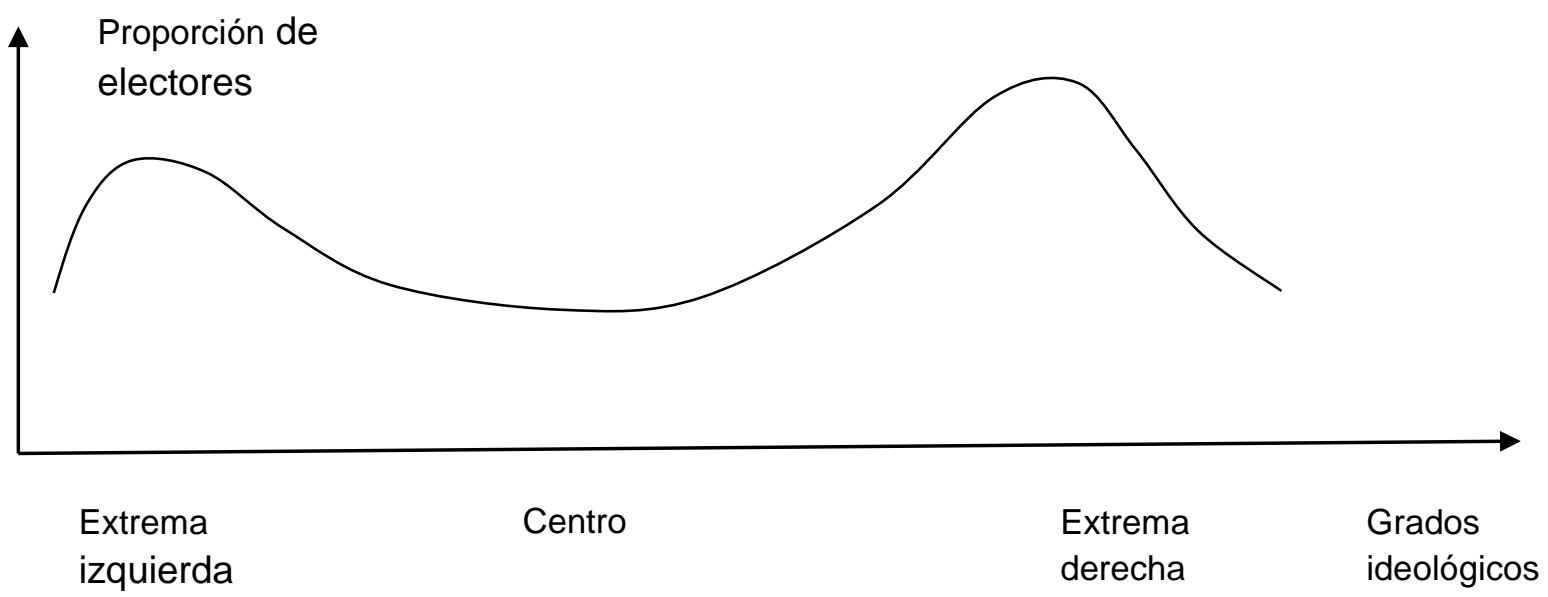

Más allá del contenido de la transacción implícita entre los electores y quienes aspiran a representarlos, está la consideración acerca de si efectivamente los contratos van a ser cumplidos. ¿Puede el gobernante engañar de continuo, desconocer el acuerdo con la colectividad y aun así perpetuarse él mismo o a su organización política? ¿Cómo puede el Public Choice abordar la interpretación de fenómenos como las demandas políticas de grupos organizados de ciudadanos 
con pretensiones limitadas a un ámbito o tema puntual (aborto, penalización de algunos delitos, reducción de precios de productos básicos, etc.), a menudo sin el auspicio de partidos políticos? Parece haberse llevado al extremo la hipótesis del ciudadano pasivo, poco informado y privado de incentivos a la luz de su función de utilidad individual. Del mismo modo que acontece con la oferta y demanda de mercancías, en el escenario democrático se presentan shocks desde las dos fuerzas: desde quienes (quizás con muy poca frecuencia) presentan novedades programáticas o formas alternativas de enfrentar las problemáticas sociales, y desde quienes ponen de relieve necesidades específicas apremiantes de la colectividad que ameritan ser atendidas. 


\section{Propuesta metodológica}

\subsection{Dinámica de la polarización política}

La primera tarea para abordar en la evaluación consiste en efectuar la caracterización del espectro político colombiano. Para ello se utiliza la convención instituida por la corriente doctrinal del Public Choice, consistente en representar el conjunto de opciones políticas como una variable continua y susceptible de ser graficada como una distribución de frecuencia. Si el número de simpatizantes o votantes por una opción política (un programa, partido, movimiento o doctrina) es mostrado en términos relativos (porcentaje del total), la representación gráfica es esencialmente la que tendría en el análisis estadístico una función de densidad de probabilidad. Como se muestra en la figura 1 de la sección anterior, si la mayoría de los electores se alinea con la opción de centro, la forma de la distribución es acampanada. La simplificación de los postulados ideológicos en una única variable acoge los tradicionales conceptos de "izquierda" y "derecha" propios de la teoría política, en los que la primera se encuentra asociada a la defensa del principio de igualdad, y la segunda es ligada a la protección del orden social, la propiedad privada y el sistema de mercado. Así, entonces, de izquierda a derecha se dispuso la siguiente secuencia de opciones políticas, según la institución que las representa:

1. Polo Democrático Alternativo ${ }^{1}$

\footnotetext{
${ }^{1}$ Para modelar el agrupamiento de electores en la contienda política de 2002 fue preciso realizar una homologación de categorías respecto a los partidos Polo Democrático y Partido Conservador, los cuales no participaron directamente. Aunque miembros afines a estas organizaciones se inscribieron como candidatos, lo hicieron bajo otros movimientos, lo cual forzó a generar una recaudación. Así pues, la categorización de facciones políticas fue la siguiente:

1 -Movimientos de izquierda "radical":Frente Social y Político Vía Alterna (Luis Eduardo Garzón); Movimiento 19 de abril (Augusto Lora)
} 


\section{Partido Liberal Colombiano}

3. Otros movimientos

4. Partido Conservador Colombiano

5. Agrupaciones denominadas "uribistas"

El rótulo "otros movimientos" contiene aquellas opciones de naturaleza socialdemócrata que propenden por una conciliación entre la libertad económica y la garantía de los derechos fundamentales de los ciudadanos. Se inscriben en esta línea el Partido Verde (hasta el proceso electoral de 2014) y las agrupaciones que como el Partido de la $U$ y Cambio Radical, en los procesos electorales de 2010 y 2014 han acogido el postulado de la llamada Tercera Vía: el mercado hasta donde sea posible y el Estado hasta donde sea necesario. Debe advertirse que estas mismas agrupaciones, antes de las elecciones de 2010 , pertenecían a la opción de derecha uribista². De este modo, queda en evidencia que representar

2 -Partido Liberal Colombiano (Horacio Serpa)

3 -Otros movimientos

4 -"Sustitutos" del Partido Conservador Colombiano: Movimiento Fuerza Colombia (Harold Bedoya); Movimiento Sí Colombia (Noemí Sanín)

5 -Agrupaciones Uribistas: Primero Colombia (Álvaro Uribe)

Al replicar el ejercicio para la elección de 2006, la conformación de categorías conllevó a considerar que, de nuevo, surge un sustituto de la alineación conservadora, en razón a que la inmensa mayoría de dirigentes del partido apoyaron la candidatura de Primero Colombia. Por afinidad entre candidato y partido, el Movimiento Nacional de Reconciliación fue tomado como asimilado al pensamiento conservador. La elección tuvo como rasgo distintivo la ostensible reducción en el número de partidos en contienda (siete frente a diez en 2002). La descripción de grupos es la siguiente:

1 -Polo Democrático Alternativo (Carlos Gaviria)

2 -Partido Liberal Colombiano (Horacio Serpa)

3 -Otros movimientos

4 -“Sustituto" del Partido Conservador Colombiano: Movimiento Nacional de Reconciliación (Álvaro Leyva)

5 -Agrupaciones uribistas: Primero Colombia (Álvaro Uribe)

2 Para mostrar este tránsito y dar cuenta de la percepción previa a las elecciones de 2010 , el Partido de la $U$ es alineado en la citada vigencia como continuador de la línea política de derecha (en 2014 se desplaza hacia el centro del espectro), mientras que Cambio Radical es asimilado en el mismo nivel del Partido Liberal, dado el origen de muchos de sus dirigentes. 
el espectro político requiere explorar la coyuntura de los partidos políticos cuya plataforma ideológica aparece fuertemente ligada a la línea de pensamiento de su caudillo o líder natural.

A su vez, se entiende por polarización un proceso por el cual la preferencia de los votantes o electores se inclina o sesga hacia los dos extremos de la distribución, de modo que la fracción de votantes en la opción de centro se reduce, dando lugar a una configuración como la representada en la figura 2 de la sección previa, correspondiente a una distribución bimodal. El proceso de cambio de distribución de unimodal a bimodal requiere una unidad de medida para la cual la teoría estadística no provee herramientas alrededor de las cuales haya consenso o amplia difusión, por lo que en la presente evaluación se adoptará un procedimiento propio mediante una modificación o adecuación del índice Herfindahl para la concentración de datos. La modificación es necesaria en razón a que los índices de concentración no distinguen posiciones de orden en las observaciones sobre las que se efectúa el cálculo. En el presente contexto, ello significa que el valor del índice calculado de forma convencional no permite identificar qué opciones políticas han ganado o perdido representación o simpatía en los electores. Un índice Herfindahl alto simplemente representaría un sesgo, independientemente de si el movimiento o partido alrededor del cual se concentra la preferencia es próximo al centro del espectro o pertenece a algún extremo.

El índice convencional es calculado mediante la expresión $H=\Sigma s^{2}$, donde $s_{i}$ en el caso en cuestión es el porcentaje de electores de cada agrupación política en una elección dada. La propuesta de modificación consiste en agregar un factor que refleje las distancias (diferencias aritméticas) entre la proporción de electores en cada zona del espectro y la proporción en la opción de centro. Si al subíndice $i$ se 
le asignan los valores 1, 2, 3, 4 y 5 para identificar las opciones ideológicas de izquierda a derecha, el índice modificado corresponderá a la expresión:

$$
H=\sum_{i=1}^{5} a b s\left(S_{i}-S_{3}\right) * S_{i}^{2}
$$

Donde abs es la función de valor absoluto.

Existe una segunda ruta de evaluación, que consiste en calcular la diferencia existente entre las frecuencias de los extremos del espectro y la frecuencia de la posición de centro, complementada por el opuesto del estadístico de asimetría de la distribución de electores ${ }^{3}$. Como es sabido, la asimetría de una distribución simétrica (como la normal, la uniforme o la t de Student) es cero, y se incrementa en valor absoluto a la par con la formación de sesgos. De esta forma, este segundo indicador calculará, para un intervalo entre dos momentos o cortes temporales, la expresión:

$\left(S_{1}+S_{5}-S_{3}\right)-a b s($ asimetría $)$

De la manera formulada, si entre dos procesos electorales la distancia entre las preferencias por las facciones políticas extremas y la facción de centro aumenta, y a la vez se mantiene estable o disminuye la asimetría, quedará evidenciado un incremento de la polarización.

\footnotetext{
${ }^{3}$ La medida de asimetría más utilizada en estadística es el coeficiente de Fisher $\mu 3 / \sigma^{3}$, donde $\mu 3$ es el tercer momento alrededor de la media y $\sigma$ es la desviación estándar. A su vez, el momento central de orden 3 es la esperanza matemática del cubo de la diferencia entre la variable evaluada y su media.
} 
Los datos a considerar se refieren al proceso electoral para la Presidencia de la República en los años 2002, 2006, 2010 y 2014. Para proveer homogeneidad, se tomaron en cuenta los resultados de la primera vuelta en aquellos casos en los que la elección contempló dos vueltas (2010 y 2014). Se prefirió este proceso electoral a otros en razón a la menor profusión de partidos o movimientos muy pequeños, cuya orientación ideológica (es decir la posición dentro del espectro) resulta difícil de identificar 4 .

\subsection{Participación política y desempeño socioeconómico}

La segunda parte de la evaluación considera la relación entre el grado de profundización de la democracia participativa y los logros en la gestión pública a los que da lugar la administración a cargo del partido o movimiento ganador de la elección. Examinar esta relación implicaría abordar diversos canales de interacción entre las organizaciones de ciudadanos y los gobernantes, como los consejos comunales o las rendiciones de cuentas, pero esta convergencia de gobernante y gobernados es apenas facial o formal, y debe complementarse con la cotidianidad de la gestión pública en procura de cumplir un programa de desarrollo. De esta forma, con espacios de encuentro o sin ellos, es razonable asumir que si un colectivo de ciudadanos es activo, inquieto o reflexivo frente a la problemática que lo rodea, expresará su opinión en las urnas y con posterioridad efectuará un seguimiento o monitoreo, así sea implícito o canalizado por los medios de comunicación bajo la forma de aceptación o preferencia del acontecer de la esfera pública.

Para propósitos del presente análisis, se utiliza la proporción de votantes respecto del censo electoral como variable que sintetiza el fenómeno de la participación.

\footnotetext{
${ }^{4}$ Incluso dentro de la elección presidencial se encontraron casos de movimientos cuyo perfil
} ideológico tuvo poca difusión, motivo por el cual fueron excluidos sus votos reportados. 
Por lo que respecta a los logros de política en cuanto a indicadores relacionados con las demandas más acuciantes de la población, debe señalarse que los recursos fiscales, según su procedencia, tienen destinaciones cualitativamente distintas. Los recursos que por derecho propio (Constitución Política y Actos Legislativos) pertenecen a una entidad territorial dentro del Sistema General de Participaciones -SGP- tienen en su mayor parte usos predefinidos en inversión social. En contraste, los ingresos corrientes de libre destinación -ICLD-, es decir, los recursos propios derivados de la actividad tributaria y de la generación de otras rentas regulares, se orientan esencialmente al funcionamiento, al cubrimiento económico del aparato burocrático. Otros tipos de ingreso, cada uno con reglas de determinación y asignación distintas, son las transferencias del sector descentralizado (empresas públicas), las operaciones de endeudamiento y las regalías.

Esta diferenciación lleva a que, por su trascendencia e impacto, sea preferible centrar la atención, para el cotejo frente a la participación política, en los recursos que un gobierno obtiene por su propia gestión y los destina a inversión social. A pesar de que una entidad territorial individualmente considerada no genera directamente los ingresos corrientes que financian el SGP, sí contribuye a que las asignaciones calculadas anualmente por el Departamento Nacional de Planeación (DNP) con información reportada de los Ministerios de los Sectores a los que se destinan las partidas, varíe a favor o en contra del departamento o municipio. Esto ocurre porque las asignaciones se ligan o atan parcialmente a metas de gestión. En los distintos componentes del SGP cabe distinguir entre un componente "inercial" (proporciones fijas) y uno variable, con el que se premia o castiga el esfuerzo propio de la administración local. En la desagregación por componentes y subcomponentes del SGP, las asignaciones por mérito o logro comprenden: 
- En el componente educación, el subcomponente de asignación por población atendida (creación de cupos).

- En el componente de agua y saneamiento, los subcomponentes población atendida, ampliación de cobertura y eficiencia.

- En el componente de propósito general, los subcomponentes de eficiencia fiscal y administrativa.

- En el componente de salud, los subcomponentes continuidad del régimen subsidiado y complemento a la prestación de servicios a la población pobre no asegurada.

De este modo, se utilizará el agregado de los conceptos mencionados como indicador de logro, ya que se trata de variables representativas de esfuerzo o de incentivo, en el sentido de que las sumas transferidas dependen de gestiones previas, como la racionalización de gastos de funcionamiento (ahorros fiscales en la operación de la burocracia local): entre más se ahonde en ella, mayor monto de transferencia es recibida.

Ahora bien, como la planificación de metas de política en los sectores que atienden las necesidades básicas procede del nivel nacional, es evidente que la elección del gobernante local no es particularmente relevante en la evaluación. De hecho, los asuntos a cargo de una administración departamental o municipal en tales esferas deben forzosamente adecuarse o compatibilizarse con las iniciativas nacionales. No obstante, desde la perspectiva de la gestión, es el gobernante local el que tiene a cargo las decisiones administrativas que permiten la utilización de recursos del SGP, lo que en algún sentido lo convierte en "agente" o "desarrollador" de los planes nacionales de gobierno en materia de atención de necesidades básicas. 
Con estas precisiones se dispuso realizar el cotejo entre la participación en la primera vuelta de la elección presidencial de 2010 y las asignaciones de SGP establecidas para la vigencia 2011 (las cuales requieren información generada en 2010 para su cálculo), efectuando ordenamientos o rankings de municipios y luego utilizando los números de posición u ordinales para calcular índices de semejanza.

Las herramientas cuantitativas para la comparación o el cotejo de series de datos son de muy variada naturaleza, y cada una presenta a partir de su mecánica de cálculos una caracterización propia del fenómeno evaluado. Así por ejemplo, los métodos de regresión enfatizan y permiten conceptuar acerca de la adecuación o proximidad de los datos a una forma funcional específica (lineal, polinómica, exponencial, logarítmica, otra). Los métodos de evaluación multicriterio, incorporados en herramientas informáticas como NAIADE (Novel Approach to Imprecise Assessment and Decision Environments) exploran la afinidad o compatibilidad entre preferencias de distintos grupos de agentes sobre un asunto de interés colectivo, en los que inicialmente no hay valoración cuantitativa explícita de las opciones sobre las que se elige, pero los ordenamientos de preferencia permiten generar relaciones de cercanía representados en dendogramas (representaciones tipo árbol). Y las técnicas de evaluación de impacto (propensity score, doble diferencia, variables instrumentales), aplicadas esencialmente a programas públicos, suelen basarse en el diseño de un contrafactual, es decir un conjunto de observaciones sobre individuos no intervenidos o afectados por la política o programa examinado. Ninguna de estas categorías de métodos aborda un problema de baja complejidad, como lo es la determinación de la sintonía o semejanza entre ordenamientos de series que no proceden de preferencias, sino de un criterio objetivo, en este caso, mediciones del nivel de participación política y mediciones de asignaciones de recursos fiscales en respuesta a logros de gestión de gobierno. Por ende, se dispuso elaborar un procedimiento propio o ad-hoc, en el que se toman los ordenamientos de entidades territoriales (municipios) y se 
aplica un indicador de semejanza a partir de la agregación de distancias entre los números de orden de cada municipio en dos series.

Si el vector $A=\left(a_{1}, a_{2}, \ldots, a_{n}\right)$ representa el ordenamiento por niveles de participación y el vector $B=\left(b_{1}, b_{2}, \ldots, b_{n}\right)$ corresponde al ordenamiento resultante de la distribución de recursos SGP "por gestión propia" per cápita, el indicador de semejanza es, formalmente, para un conjunto de $n$ municipios:

$$
S=(1 / n) * \sum a b s\left(a_{i}-b_{j}\right)
$$

Donde abs es la función de valor absoluto, i es la posición de un municipio en el ordenamiento $\mathrm{A}, \mathrm{y} \mathrm{j}$ es la posición del mismo municipio en el ordenamiento $\mathrm{B}$. Entre mayor valor posea el indicador, menos semejanza existirá entre los ordenamientos.

Dado que un departamento con muchos municipios tenderá a tener distancias ( $\mathrm{a}_{\mathrm{i}}$ $b_{j}$ ) más grandes que las generadas en un departamento pequeño, es necesario un procedimiento adicional de estandarización o transformación. Se dispuso convertir los números de posición en valores relativos de acuerdo a la localización del valor de la variable entre el máximo y el mínimo obtenidos en el agregado de municipios, trasladando luego la fracción generada a una escala de 0 a 10 .

Asúmase, por ejemplo, que para un departamento la variable $Y_{1}$ (participación política) posee, en sus cinco municipios, los valores 2, 3, 6, 7 y 9; y para la variable $Y_{2}$ (un subcomponente cualquiera del SGP per cápita) los valores en los mismos municipios son: 4, 5, 8, 9 y 10 . 
Al sustituir los valores ordinales por la convención descrita, el cálculo del indicador de semejanza operaría como se describe en la tabla adjunta.

Tabla 1. Simulación de cálculo del indicador de semejanza

\begin{tabular}{|c|c|c|c|c|c|}
\hline \multicolumn{6}{|c|}{ Participación política } \\
\hline Valor & Max & Min & Max-Min & Valor-Min & $\begin{array}{c}a_{i}=10^{*}(\text { valor- } \\
\text { Min)/(Max-Min) }\end{array}$ \\
\hline 2 & & & & 0 & 0,00 \\
\hline 3 & & & & 1 & 1,43 \\
\hline 6 & 9 & 2 & 7 & 4 & 5,71 \\
\hline 7 & & & & 5 & 7,14 \\
\hline 9 & & & & 7 & 10,00 \\
\hline \multicolumn{6}{|c|}{ Subcomponente del SGP } \\
\hline Valor & $\operatorname{Max}$ & Min & Max-Min & Valor-Min & $\begin{array}{c}b_{j}=10^{*} \text { (valor- } \\
\text { Min)/(Max-Min) }\end{array}$ \\
\hline 4 & & & & 0 & 0,00 \\
\hline 5 & & & & 1 & 1,67 \\
\hline 8 & 10 & 4 & 6 & 4 & 6,67 \\
\hline 9 & & & & 5 & 8,33 \\
\hline 10 & & & & 6 & 10,00 \\
\hline
\end{tabular}

\begin{tabular}{cc}
\hline & Indicador de semejanza \\
\hline $\operatorname{abs}\left(\mathrm{a}_{\mathrm{i}}-\mathrm{b}_{\mathrm{j}}\right)$ & \\
0,00 & $\mathrm{~S}=(1 / 5)^{\star} \Sigma$ abs $\left(\mathrm{a}_{\mathrm{i}}-\mathrm{b}_{\mathrm{j}}\right)$ \\
0,24 & $=(1 / 5)^{\star} 2,38$ \\
0,95 & $=0,476$ \\
1,19 & \\
0 & \\
\hline
\end{tabular}

Fuente: elaboración propia. 
Como última modificación, dada la ocurrencia de situaciones atípicas en cuanto a ratios de participación en las elecciones, sobre las cuales organismos como la Misión de Observación Electoral -MOE- han indicado que pueden encubrir situaciones de anormalidad, casos de delitos electorales, presiones al elector, restricciones a la movilización o similares, se decidió separar del ejercicio los municipios con el $2 \%$ de mayores y menores participaciones a nivel de todo el país $^{5}$. Ello conduce a que, de 1.102 territorios entre municipios y distritos, sean utilizados los datos de 1.058 en la evaluación.

Al tener en cuenta los valores extremos que puede tomar el indicador $\mathrm{S}$ (cero y diez), pueden establecerse tres rangos para $S$ con su correspondiente interpretación:

- Si S se localiza entre 0 y 3,33, la coincidencia entre dos series ordenadas A y B se cataloga como elevada.

- Si S se localiza entre 3,34 y 6,66, la coincidencia es de nivel intermedio.

- Si S se localiza entre 6,67 y 10, existe bajo nivel de coincidencia, es decir que las series tienden a mostrar ordenamientos contrarios: si $Y_{1}$ aumenta, $\mathrm{Y}_{2}$ disminuye y viceversa.

5 Mapas de riesgo electoral 2002-2006. Misión de Organización Electoral, en http://www.moe.org.co. La institución distingue niveles variados de situaciones atípicas. 


\section{Resultados de la evaluación}

\subsection{Cambios en el espectro político en Colombia 2002-2014}

A partir de la estadística de votos válidos según lo expuesto en la sección 2.1 se obtuvieron los siguientes valores para las proporciones de votantes:

Tabla 2. Proporciones de votantes en el espectro político colombiano 2002-2014

\begin{tabular}{|c|c|c|c|c|c|}
\hline \multirow{2}{*}{$\begin{array}{l}\text { Proceso } \\
\text { electoral }\end{array}$} & \multicolumn{5}{|c|}{ Opción política (desde izquierda hasta derecha) } \\
\hline & 1 & 2 & 3 & 4 & 5 \\
\hline 2002 & $6,39 \%$ & $32,50 \%$ & $0,50 \%$ & $5,93 \%$ & $54,68 \%$ \\
\hline 2006 & $22,52 \%$ & $12,10 \%$ & $1,63 \%$ & $0,00 \%$ & $63,75 \%$ \\
\hline 2010 & $9,33 \%$ & $14,80 \%$ & $21,96 \%$ & $6,26 \%$ & $47,66 \%$ \\
\hline 2014 & $16,18 \%$ & $0,00 \%$ & $36,16 \%$ & $16,51 \%$ & $31,15 \%$ \\
\hline
\end{tabular}

Fuente: Cálculos propios / Datos extraídos de la Registraduría Nacional del Estado Civil, sitio web.

Con base en las cifras calculadas en la tabla pueden generarse los valores del índice $\mathrm{H}$ modificado y el indicador de distancias y asimetría como sigue:

Tabla 3. Indicadores de polarización política

\begin{tabular}{ccc}
\hline Proceso electoral & Índice H modificado & $\begin{array}{c}\text { Indicador de distancias y } \\
\text { asimetría }\end{array}$ \\
\hline 2002 & 0,1962 & $-0,45$ \\
2006 & 0,2646 & $-0,78$ \\
2010 & 0,0616 & $-1,23$
\end{tabular}


Fuente: elaboración propia.

En el 2006, la polarización se exacerba y con ello la dificultad para la consecución de consensos sociales alrededor de los programas y proyectos derivados de los lineamientos de política. Ello, a la luz de lo expuesto en el marco teórico, puede ser visto como un alejamiento de las acciones de gobierno frente a la posición óptima de la función de bienestar social. Claramente, si una porción importante de individuos se encuentra en desacuerdo con el régimen vigente, su utilidad declina, y así mismo la del agregado social.

Salvo la coyuntura de 2006 en el índice H modificado, hay consistencia en el comportamiento conjunto de las dos mediciones, en el sentido de que se observa una tendencia decreciente en el grado de polarización, indistintamente del signo algebraico de los resultados obtenidos. Por definición, $\mathrm{H}$ es un valor positivo, mientras que si la diferencia entre proporciones de votantes "extremos" y "medios" no compensa la asimetría, entonces resulta un valor negativo, como ocurrió con los datos utilizados. A comienzos de siglo, en la elección de 2002, era muy notable la agrupación de votantes en las posiciones ideológicas 2 y 5. En 2006, crece la acumulación de votantes en las posiciones 1 y 5 , pero mucho más en esta última, por lo que la asimetría también crece, de modo que la medición por el segundo indicador sigue siendo negativa. En 2010, comienza a gestarse un cambio sustancial en el espectro, debido al posicionamiento de la opción de centro, que el período siguiente, en 2014, pasa a ser la ideología dominante, mayoritaria. 
Gráficos 1-4. Resultados de procesos electorales 2002 a 2014 - Presidencia
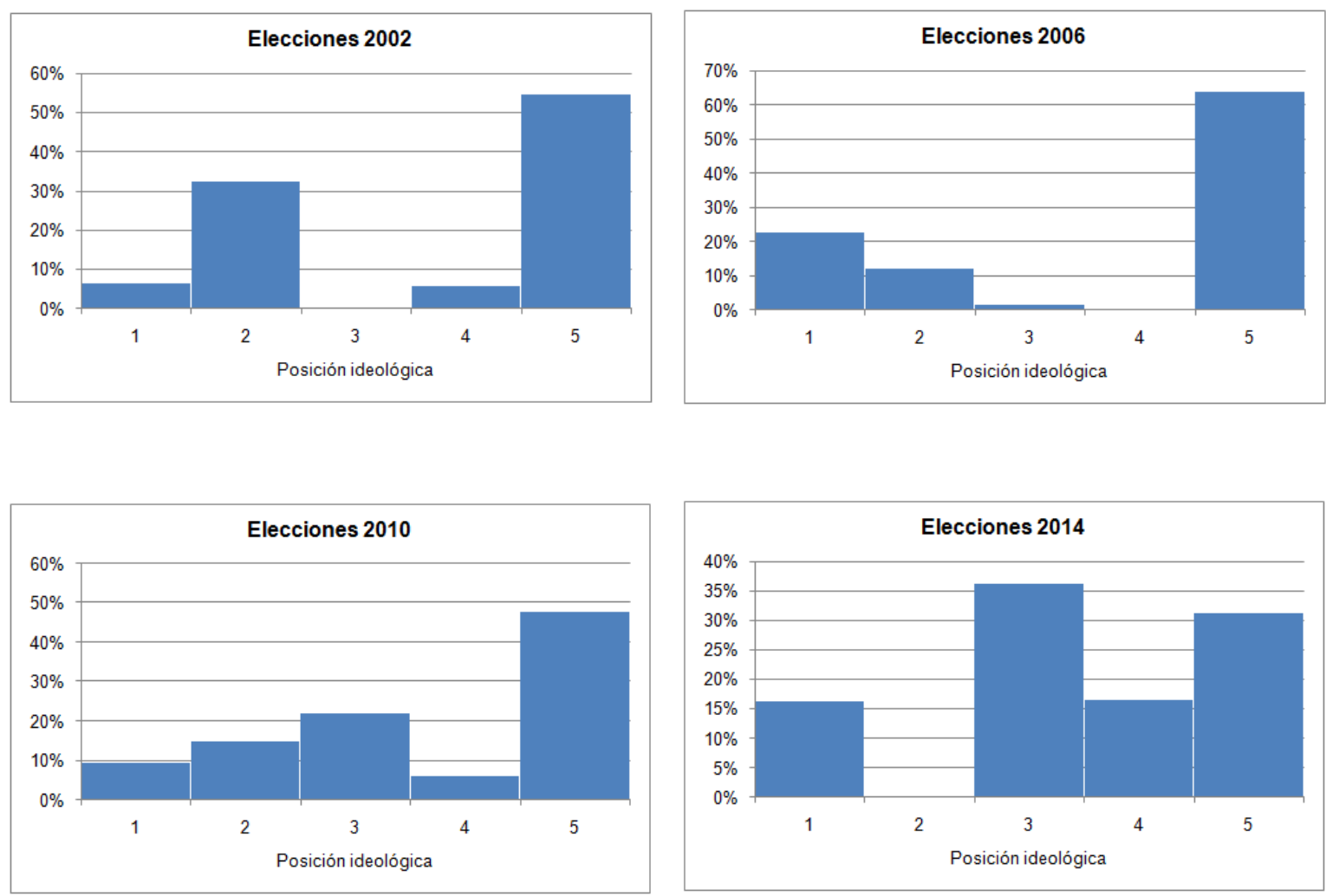

Fuente: Cálculos propios / Datos extraídos de la Registraduría Nacional del Estado Civil, sitio web.

En consecuencia, el nivel de confrontación que hoy en día se asume o interpreta entre facciones políticas no se ha reflejado en las urnas, y puede asociarse más a la conveniencia o inconveniencia de los procedimientos encauzados por la actual Administración hacia un eventual cese de la violencia armada, pero no se vincula a otros elementos o variables del sistema social (pobreza, empleo, salud, corrupción) que presumiblemente guían con más fuerza la decisión del votante en su elección de gobernante.

\subsection{Cotejo entre participación política y gestión pública municipal}


Al aplicar el procedimiento ideado sobre la participación electoral y los subcomponentes del SGP indicados en la sección 2.2, se encontró inicialmente que, de los municipios considerados como distorsionantes por su participación electoral muy alta o muy baja, la gran mayoría del primer grupo (16 municipios de los 22 excluidos) pertenece a Cundinamarca, y más concretamente a las vecindades de Bogotá, D.C. (Anexo). Este resultado es sugestivo: o existe una alta capacidad de los dirigentes de la zona para incidir sobre las decisiones de los pobladores, o la influencia sobre los electores procede de la propia capital del país, lo cual es verosímil considerando que habitantes del Distrito Capital poseen propiedades raíces en estos municipios y son de su interés las políticas desarrolladas sobre estas entidades territoriales. Otros municipios excluidos por alta participación se sitúan en la Región Oriental, siendo proclives a la alta recepción de regalías (cuatro municipios en Casanare y Castilla Nueva en Meta, un municipio caracterizado por la calidad en sus inversiones sociales).

El segundo grupo incluye, por su parte, municipios en su mayoría percibidos como de bajo desarrollo relativo, desatendidos por el Estado, con problemas o antecedentes de perturbación del orden público (Bojayá en Chocó, Miraflores en Guaviare) o en zonas de difícil acceso. De los 22 municipios excluidos, 12 están en 3 departamentos de la Región Pacífica (Chocó, Cauca y Nariño) y el empalme de esta zona con la Amazonia (Caquetá).

El paso siguiente, la tabulación de los valores asignados de SGP por componentes y subcomponentes, mostró que estos aplican a todo el universo de municipios y distritos, con una excepción: el concepto de población atendida en educación. Esta partida es asignada solamente a un grupo de entidades territoriales certificadas (muchas de ellas capitales de departamento), es decir, que han logrado un nivel alto de logros en la materia. 
La caracterización de los montos per cápita es como sigue:

La variable que en promedio muestra la mayor dimensión en pesos por habitante es precisamente la partida para población atendida en educación, con un valor máximo de \$421.947 en Quibdó (Chocó), y un mínimo de cero para los municipios no certificados (999 de los 1.058 evaluados).

En segundo lugar, se ubican los conceptos asociados al componente salud. El monto más elevado, \$227.154 por habitante, lo recibió San Andrés de Sotavento (Córdoba). La menor cifra se encontró, paradójicamente, en el mismo departamento, en San José de Uré $(\$ 5.298)$.

La tercera variable en orden promedio de magnitud fue el agregado de conceptos de la asignación para propósito general. La cifra más alta correspondió a Sativasur, Boyacá, con la cifra puntual más alta dentro de las variables utilizadas: $\$ 537.894$ por habitante. En cuanto al valor mínimo, 26 municipios no tuvieron asignación de recursos.

Finalmente se encuentra la asignación para agua y saneamiento. En ella, el mayor municipio receptor fue San José de Uré, en Córdoba, con $\$ 55.649$ por habitante. Ello es señal de las fuertes distinciones entre los criterios para cada subcomponente del SGP: un municipio localizado estrictamente en el último puesto por un subcomponente es a la vez el primero en un subcomponente de otra categoría. El menor registro, de cero, correspondió a Mapiripán (Meta).

Estructuradas las respectivas tablas, se calculó, para cada municipio y variable, la distancia relativa (Valor-Min)/(Min-Max), se multiplicó por 10 para generar la escala de $\mathrm{S}$ y se establecieron los valores absolutos de las diferencias frente al 
mismo cálculo sobre la variable de participación. El resultado final del cotejo aparece en la siguiente tabla:

Tabla 4. Cálculo de indicadores de semejanza

\begin{tabular}{lcrrrr}
\hline Departamento & S1 & \multicolumn{2}{c}{ S2 } & S3 & \multicolumn{2}{c}{ S4 } & Promedio \\
\hline Amazonas & N.A. & 0,00 & 0,00 & 10,00 & 3,33 \\
Antioquia & 4,35 & 2,30 & 3,95 & 2,80 & 3,35 \\
Arauca & N.A. & 4,43 & 4,29 & 5,71 & 4,81 \\
Atlántico & 5,83 & 3,87 & 3,65 & 3,00 & 4,09 \\
Bolívar & 5,87 & 3,52 & 4,98 & 1,87 & 4,06 \\
Boyacá & 6,24 & 3,66 & 5,15 & 2,58 & 4,41 \\
Caldas & 5,04 & 2,81 & 4,00 & 2,89 & 3,69 \\
Caquetá & 3,08 & 2,83 & 2,58 & 2,92 & 2,85 \\
Casanare & 7,00 & 3,93 & 6,60 & 3,53 & 5,27 \\
Cauca & 3,79 & 2,21 & 3,41 & 3,79 & 3,30 \\
Cesar & 5,76 & 2,92 & 4,04 & 3,44 & 4,04 \\
Chocó & 3,96 & 3,46 & 3,15 & 3,77 & 3,59 \\
Córdoba & 4,87 & 3,77 & 3,40 & 2,20 & 3,56 \\
Cundinamarca & 5,36 & 3,10 & 4,61 & 4,03 & 4,28 \\
Guajira & 7,15 & 3,31 & 6,00 & 5,38 & 5,46 \\
Guaviare & N.A. & 1,00 & 4,00 & 7,67 & 4,22 \\
Huila & 5,46 & 3,03 & 4,24 & 2,35 & 3,77 \\
Magdalena & 5,37 & 2,60 & 3,63 & 2,63 & 3,56 \\
Meta & 5,89 & 2,36 & 4,71 & 3,36 & 4,08 \\
Nariño & 5,03 & 2,02 & 3,35 & 2,00 & 3,10 \\
Norte de & 5,55 & 3,20 & 4,73 & 2,78 & 4,06 \\
Santander & N.A. & 3,15 & 3,38 & 5,00 & 3,85 \\
Putumayo & 4,67 & 4,42 & 5,08 & 5,08 & 4,81 \\
Quindío & & & & &
\end{tabular}




\begin{tabular}{lrrrrr} 
Risaralda & 5,07 & 3,71 & 5,79 & 4,36 & 4,73 \\
San Andrés & N.A. & 10,00 & 10,00 & 10,00 & 10,00 \\
Santander & 4,73 & 2,53 & 3,49 & 2,43 & 3,30 \\
Sucre & 6,46 & 2,88 & 5,96 & 3,12 & 4,61 \\
Tolima & 5,62 & 2,49 & 4,15 & 2,83 & 3,77 \\
Valle del Cauca & 3,90 & 2,14 & 3,62 & 2,69 & 3,09 \\
Vaupés & N.A. & 7,00 & 8,33 & 6,67 & 7,33 \\
Vichada & N.A. & 1,67 & 3,67 & 9,00 & 4,78 \\
Promedio & 5,25 & 3,24 & 4,45 & 4,19 & \\
\hline
\end{tabular}

Fuente: elaboración propia.

N.A. $=$ No aplica.

En la tabla aparecen siete registros de "no aplica" en razón a variables que tienen distinto grado de agrupación para los corregimientos de algunos de los antiguos territorios nacionales, lo cual impidió los cálculos.

Al clasificar por rangos de valores, 39 de los 124 indicadores individuales $(31,45 \%)$ pertenecen al criterio de coincidencia moderada a elevada; 68 registros $(54,84 \%)$ pertenecen a la zona de indefinición (entre coincidencia y discrepancia), y 10 $(8,1 \%)$ muestran de moderada a alta discrepancia, es decir, sentido opuesto en los ordenamientos. Desde ese punto de vista, los datos se inclinan hacia la coincidencia, aunque de una manera más bien leve.

Por categorías, en cambio, se encuentran diferencias sustanciales: el mayor grado de coincidencia se presenta en el componente de agua y saneamiento, con promedio de 3,24 y 20 de sus 31 registros con valores de coincidencia de moderada a alta. Es seguido por el componente de salud (4,19 en promedio), el de propósito general $(4,45)$ y el de educación $(5,25)$, los tres en la zona de 
indefinición. Es destacable que sean precisamente las variables asociadas a los derechos biológicos (agua y salud) las que muestren el mejor desempeño. Ello respalda la tesis de que la provisión de bienes y servicios esenciales, al impulsar la calidad de vida, estimula la búsqueda de pretensiones sociales de naturaleza distinta, como la participación política.

Es posible también realizar la lectura alterna, es decir mediante la comparación de promedios por departamento. De menor a mayor valor, obtienen un desempeño satisfactorio en términos del grado de coincidencia los departamentos de Caquetá $(2,85)$, Valle $(3,09)$, Nariño $(3,10)$, Santander $(3,30)$, Cauca $(3,30)$ y Amazonas $(3,33)$. Antioquia se sitúa muy cerca del límite de categoría, con un registro de 3,35 . Aunque parezca que hay heterogeneidad, en los departamentos citados se hallan tres de las mayores zonas urbanas del país (Cali, Bucaramanga y Medellín), sin considerar que Bogotá, D.C. debió ser excluida en razón a que con una única observación no puede generarse el indicador respectivo. Aparte de ello, la Región Pacífica tiene gran protagonismo en el ordenamiento, lo que puede denotar afinidad frente a problemáticas de acceso deficiente a servicios, presencia de grupos armados ilegales y difícil acceso vial.

Al otro lado del espectro se sitúan Arauca $(4,81)$, Quindío (4,81), Casanare $(5,27)$, Guajira $(5,46)$, Vaupés $(7,33)$ y San Andrés $(10,0)$. Los dos casos de inclinación a la discrepancia son realmente consecuencia de la cantidad mínima de municipios (dos en el archipiélago y tres en Vaupés), de manera que descontándolos, no quedarían casos de incompatibilidad frente al criterio de coincidencia. Es de interés la presencia de Arauca y Casanare, en el sentido de que la generación de rentas petroleras no ha inducido un afinamiento entre la provisión de servicios básicos y el interés por alcanzar logros de gestión. 


\section{Conclusiones}

El espectro político colombiano ha estado sujeto a cambios notables en lo corrido del siglo XXI, partiendo de una alta concentración en las posiciones políticas extremas y evolucionando luego hacia un acercamiento a la posición del votante medio. Con este resultado, obtenido a partir del índice de Herfindahl reformado y el indicador de distancias y asimetría, queda descartada la hipótesis de reforzamiento del fenómeno de polarización. En apariencia, las diferencias de opinión en la ciudadanía versan sobre aspectos instrumentales del ejercicio de la política más que sobre elementos del sistema social alrededor de los que se decida verdaderamente un proceso electoral.

En lo referente a la interacción entre los niveles de participación y los resultados de gestión reflejados en la asignación de recursos del SGP, el procedimiento de evaluación establecido dio lugar a una validación leve del postulado de sintonía entre los ordenamientos de municipios. Esto significa que en promedio, la participación es acompañada en alguna medida por mejoras en la gestión de recursos, al menos a la luz de los estándares de logro utilizados por el gobierno nacional para el cálculo de las transferencias en educación, agua y saneamiento básico, propósito general y salud.

Por regiones, la mayor sintonía o afinidad entre ordenamientos, es decir, las zonas donde mejor se cumple la interrelación de participación y gestión gubernamental ocurre allí donde se ubican las grandes ciudades, lo cual puede asociarse al sentido de cultura política y control de las acciones de la administración municipal. Por componentes, el mayor grado de sintonía ocurre alrededor de las necesidades esenciales (agua y saneamiento, salud), lo cual es compatible con la mayor utilidad marginal de los bienes básicos frente a los suntuarios según la teoría 
microeconómica, y que implícitamente condicionaría la elección entre alternativas o mercaderías políticas en el sentido descrito por el Public Choice.

De los anteriores resultados, y de la investigación en sí misma, surgen recomendaciones en dos sentidos. De una parte, se hace necesaria una profundización de la cultura política en las regiones periféricas de la geografía nacional, tanto por la vía de la participación formal en los procesos democráticos como por la vía de los mecanismos de control ciudadano, bajo la guía o supervisión de los organismos públicos que ejercen vigilancia a la gestión pública. Esto puede redundar a mediano plazo en un mejor posicionamiento de estos municipios en los ordenamientos de SGP, de tal modo que entre a operar un círculo virtuoso de gestión favorable y recursos para atender las necesidades esenciales de los pobladores.

De otro lado, se hace manifiesto a la precariedad instrumental que tiene para efectos de la validación de sus postulados, a la luz de la evidencia empírica, la teoría Public Choice. A pesar de la riqueza de las herramientas de representación matemática sobre la economía neoclásica, no se han efectuado adaptaciones desde el enfoque marginalista hacia evaluaciones de fenómenos de elección política en el marco de la democracia formal. Es pues, un terreno sobre el cual queda un amplio margen de maniobra para efectuar aportaciones metodológicas que conduzcan a una valoración, por parte de la comunidad académica, de la pertinencia de la teoría de la elección pública para interpretar el fenómeno de la actividad política. 


\section{Anexo. Municipios excluidos de la evaluación por sus niveles atípicos de participación política en 2011}

\begin{tabular}{llc}
\hline Departamento & \multicolumn{1}{c}{ Municipio } & $\begin{array}{c}\text { Participación electoral } \\
\text { (Votantes / Censo) }\end{array}$ \\
\hline Cundinamarca & El Rosal & $74,9 \%$ \\
Cundinamarca & Sopo & $71,6 \%$ \\
Cundinamarca & Tocancipá & $71,5 \%$ \\
Cundinamarca & Bojacá & $71,1 \%$ \\
Casanare & Chámeza & $71,0 \%$ \\
Cundinamarca & Mosquera & $70,9 \%$ \\
Cundinamarca & Tabio & $70,8 \%$ \\
Cundinamarca & Gachancipá & $70,8 \%$ \\
Santander & Vetas & $70,3 \%$ \\
Cundinamarca & Facatativá & $70,2 \%$ \\
Cundinamarca & Madrid & $69,8 \%$ \\
Cundinamarca & Tenjo & $69,6 \%$ \\
Cundinamarca & Villapinzón & $69,4 \%$ \\
Casanare & Monterrey & $69,4 \%$ \\
Cundinamarca & Suesca & $69,1 \%$ \\
& Castilla La & $68,8 \%$ \\
Meta & Nueva & $68,7 \%$ \\
Casanare & Aguazul & $68,5 \%$ \\
Casanare & Tauramena & $68,5 \%$ \\
Cundinamarca & Funza & $68,5 \%$ \\
Cundinamarca & Cajicá & $68,0 \%$ \\
Cundinamarca & Cogua & $68,0 \%$ \\
Cundinamarca & Sesquilé &
\end{tabular}




\section{Anexo (continuación)}

\begin{tabular}{|c|c|c|}
\hline Departamento & Municipio & $\begin{array}{c}\text { Participación electoral } \\
\text { (Votantes / Censo) }\end{array}$ \\
\hline Antioquia & Toledo & $22,2 \%$ \\
\hline Antioquia & Briceño & $21,7 \%$ \\
\hline Caquetá & La Montanita & $21,4 \%$ \\
\hline Chocó & Riosucio & $20,9 \%$ \\
\hline Amazonas & La Pedrera & $20,6 \%$ \\
\hline Nariño & Ricaurte & $20,6 \%$ \\
\hline Cauca & Argelia & $20,5 \%$ \\
\hline Nariño & $\begin{array}{l}\text { Santa Bárbara } \\
\text { (Iscuande) }\end{array}$ & $20,0 \%$ \\
\hline Cauca & Almaguer & $20,0 \%$ \\
\hline Caquetá & $\begin{array}{l}\text { Cartagena Del } \\
\text { Chairá }\end{array}$ & $20,0 \%$ \\
\hline Amazonas & Puerto Alegría & $19,7 \%$ \\
\hline Chocó & Juradó & $18,9 \%$ \\
\hline Caquetá & Solano & $18,4 \%$ \\
\hline Amazonas & La Victoria & $18,2 \%$ \\
\hline Chocó & $\begin{array}{l}\text { Bojayá } \\
\text { (Bellavista) }\end{array}$ & $18,1 \%$ \\
\hline Vaupés & $\begin{array}{l}\text { Buenos Aires } \\
\text { (Pacoa) }\end{array}$ & $17,9 \%$ \\
\hline Antioquia & Anorí & $17,3 \%$ \\
\hline Antioquia & Ituango & $17,3 \%$ \\
\hline Antioquia & Campamento & $17,0 \%$ \\
\hline Guaviare & Miraflores & $16,4 \%$ \\
\hline Guainía & $\begin{array}{l}\text { Barranco } \\
\text { Minas }\end{array}$ & $16,2 \%$ \\
\hline Chocó & $\begin{array}{l}\text { El Litoral del } \\
\text { San Juan }\end{array}$ & $16,1 \%$ \\
\hline
\end{tabular}




\begin{tabular}{lll} 
La Guajira & Manaure & $15,7 \%$ \\
Caquetá & Milán & $15,7 \%$ \\
Guainía & San Felipe & $15,5 \%$ \\
Cauca & Lopez (Micay) & $14,6 \%$ \\
Vichada & Cumaribo & $13,0 \%$ \\
Guainía & Mapiripana & $12,4 \%$ \\
Guainía & Cacahual & $12,3 \%$ \\
& Puerto & $11,7 \%$ \\
Guainía & Colombia & $7,8 \%$ \\
La Guajira & Uribia & $4,2 \%$ \\
Amazonas & Mirití Paraná & \\
\hline
\end{tabular}

Fuente: Cálculos propios / Datos extraídos de la Registraduría Nacional del Estado Civil, sitio web. 


\section{Referencias bibliográficas}

Arrow, K. J. (1974). Una dificultad en el concepto de bienestar social. En Arrow y Scitovsky (comps.), Ensayos sobre la economía del bienestar l, pp. 151-171. México: Fondo de Cultura Económica.

Blanchard, O. J. y Fischer, S. (1989). Lectures on Macroeconomics. Cambridge: The MIT Press.

Dahl, R. (1956). A Preface to Democratic Theory. Chicago: University of Chicago Press.

Edgeworth, F. Y. (1879). The Hedonical Calculus. Mind. Volumen 4, pp. 394-408.

González, Jorge I. (1998). Arrow: la elección, los valores y la ideología del mercado, en Cuadernos de Economía, (28), pp. 36-39.

González García, J. (1992). Crítica de la teoría económica de la democracia. En González y Quesada Castro, F. (coords.), Teorías de la democracia, pp. 312-313, Barcelona: Anthropos.

Lange, O. (1974).Los fundamentos de la economía del bienestar. En Arrow y Scitovsky (comps.), Ensayos sobre la economía del bienestar I, pp. 39-50. México: Fondo de Cultura Económica.

Loyola Campos, J. (2011). La superación de la "paradoja de la (no) participación" electoral sobre bases racionales: ¿qué modelo es más adecuado? Recuperado de: 
http://www.revistaakademeia.cl/wp/wp-content/uploads/2011/12/La-superacion-dela-paradoja-de-la-no-participación-electoral-sobre-bases-racionales.pdf

Lucas, R. E. (1975). An equilibrium model of the business cycle, Journal of Political Economy, 83, pp. 15-18.

Muguerza, J. (1992). Ética y comunicación. (Una discusión del pensamiento éticopolítico de Jürgen Habermas, en González y Quesada Castro, F. (coords.), Teorías de la democracia, pp. 139-141. Barcelona: Anthropos.

Quesada Castro, F.(1992). C.B. Macpherson. De la teoría política del individualismo posesivo a la democracia participativa. En González y Quesada Castro, F. (coords.), Teorías de la democracia, pp. 297. Barcelona: Anthropos.

Sen, Amartya (1976). Elección colectiva y bienestar. Madrid: Alianza Editorial. 